\title{
Comparação da Influência entre Tempos de Polimerização em Resinas Compostas Polimerizadas com LED e Luz Incandescente
}

\author{
Michele P. M. Ulhoa, Lúcio R. S. Santana, Eduardo C. Bianchi, Carlos E. D. Cruz, Paulo R. Aguiar \\ Faculdade de Engenharia de Bauru, UNESP
}

\author{
César A. de Freitas, Márcia F. A. de Freitas \\ Faculdade de Odontologia de Bauru, USP
}

\begin{abstract}
Resumo: O propósito deste trabalho é fazer uma comparação entre resinas poliméricas dentárias, polimerizadas por aparelhos baseados em lâmpada halógena e diodo emissor de Luz (LED), utilizando-se o método do disco retificado aperfeiçoado para odontologia e os respectivos valores de microdureza. Foram realizados testes em amostras de resinas compostas de 5 diferentes marcas, polimerizadas a tempos de 10,20 e $40 \mathrm{~s}$, pelos dois aparelhos. A análise estatística dos valores de microdureza e agressividade permitiu concluir que estatisticamente não há correlação entre essas propriedades. Na análise de microdureza, a heterogeneidade característica do material implicou em resultados com valores de desvio padrão relativamente altos, de forma que não foi encontrada diferença estatística entre as amostras avaliadas. Na análise estatística dos ensaios baseados no método do disco retificado, a resina que apresentou maior desgaste nos ensaios, foi a Tetric Ceram, polimerizada pelo aparelho de LED por $10 \mathrm{~s}$, cujo valor médio de agressividade obtido foi $0,170 \mathrm{~mm}^{3} / \mathrm{N} . \mathrm{m}$. A resina que sofreu menor desgaste foi a Charisma, polimerizada por Lâmpada Incandescente, por um tempo de $20 \mathrm{~s}$, cuja média dos valores de agressividade foi $0,057 \mathrm{~mm}^{3} / \mathrm{N} . \mathrm{m}$.
\end{abstract}

Palavras-chave: Resina composta, desgaste abrasivo, métodos de avaliação, microdureza.

\section{Comparison of the Influence of Curing Times Applied to Composite Resins Cured with LED and Incandescent Light}

Abstract. The purpose of this work was to compare polymeric dental resins cured with halogen lamp and with light emission diode (LED) devices, using the grinding disk method customized for dentistry and the corresponding microhardness values. Tests were carried out on resin samples of five brands, which were cured for 10, 20 and $40 \mathrm{~s}$ with the two devices. The analysis of microhardness and aggressiveness has allowed us to conclude that there is no correlation between these properties. In Microhardness tests, the material heterogeneity has produced relative high standard deviation values and has not shown statistical differences between the analyzed samples. In the statistical analysis for the results with the grinding disk method, the resin with highest wear was the Tetric Ceram, cured with LED device for $10 \mathrm{~s}$ (aggressiveness average $0.170 \mathrm{~mm}^{3} / \mathrm{N} . \mathrm{m}$ ). The composite resin with least wear was Charisma, cured with halogen lamp device for $20 \mathrm{~s}$ (aggressiveness average $0.057 \mathrm{~mm}^{3} / \mathrm{N} . \mathrm{m}$ ).

Keywords: Composite resin, abrasive wear, evaluation methods, microhardness.

\section{Introdução}

O homem está sempre em busca de melhores condições de vida. A valorização da estética pela sociedade atual é uma delas, resultando em avanços no desenvolvimento de novos materiais sintéticos na Odontologia. Dentro destes avanços as novas fórmulas de resinas compostas fotopolimerizáveis, as quais são amplamente utilizadas como materiais restauradores para dentes anteriores e posteriores, são as que mais se destacam, principalmente em substituição a amalgama.

Dentre os vários fatores que influenciam aos dentistas a utilizarem constantemente estas resinas, estão a facilidade no manuseio e uma grande quantidade de cores, possibilitando ficarem praticamente imperceptíveis após sua aplicação.

Seria ideal que estes materiais restauradores se desgastassem de modo semelhante ao material dentário (dente), mas não é isso que ocorre. $\mathrm{O}$ fator limitante do uso das resinas compostas é a deterioração que ocorre quando estes materiais são utilizados onde estão sujeitos ao desgaste oclu$\mathrm{sal}^{[1]}$. O desgaste tem sido analisado em um enorme número de estudos clínicos, e vários tipos de testes têm sido desenvolvidos para analisá-lo nas resinas compostas e também em outros materiais restauradores. Uma análise confiável da

Autor para correspondência: Eduardo C. Bianchi, Departamento de Engenharia Mecânica, Faculdade de Engenharia de Bauru, Universidade Estadual Paulista (UNESP), Caixa postal 473, CEP: 17033-360, Bauru, SP, Brasil. E-mail: bianchi@feb.unesp.br 
resistência ao desgaste abrasivo deve auxiliar no desenvolvimento e melhora das resinas compostas, pois fornecerá uma forma de análise rápida destes produtos, sem a necessidade de aguardar os resultados dos demorados ensaios clínicos.

Existem vários fatores que implicam neste desgaste abrasivo, como escovação, mastigação, as características da resina, o preparo cavitário bem como as condições de desgaste a que são submetidas.

O mercado odontológico oferece uma ampla gama de opções para a escolha do material de trabalho aos profissionais ligados à esta área, devido à grande velocidade dos avanços tecnológicos e a competição existente nos diversos ramos. Assim, torna-se interessante, testes que possam lhes garantir uma maior confiabilidade na tecnologia que estão utilizando.

De acordo com a literatura, para a realização das análises do desgaste de resinas compostas são apresentados métodos clínicos (in vivo) e métodos laboratoriais (in vitro). O método clínico in vivo consiste em realizar restaurações em um determinado número de pacientes e, após certo período de tempo, normalmente bastante longo, cerca de 2 a 6 anos, analisar o desgaste ocorrido na resina. Neste método, existe a falta de controle sobre variáveis importantes como a força empregada na mastigação, a dieta ou fatores biológicos (bucais), que limitam o estudo clínico e em consequiência, o estudo do desgaste dos materiais dentários.

Já nos testes laboratoriais esses inconvenientes são minimizados. Dessa forma torna-se interessante realizar ensaios in vitro, que é um método que propõe ensaios laboratoriais e pode reduzir o tempo e o custo das análises com as resinas para avaliar seu comportamento, suas características e eficiência contra o desgaste abrasivo, entre outras variáveis, buscando resultados precisos, porém mais rápidos que os testes in vivo.

Bianchi et al. ${ }^{[2]}$, com o objetivo de realizar estudos de desgaste em resinas poliméricas dentárias, modificaram o método do disco retificado de Coelho ${ }^{[3]}$, adaptando-o para a avaliação de materiais restauradores odontológicos.

Para a confecção de corpos de provas para a realização de ensaios, as resinas compostas são polimerizadas com aparelhos de fotopolimerização. Kurachi et al. ${ }^{[4]}$, em seu estudo, diz que com um maior desenvolvimento dos instrumentos de fotopolimerização baseados em LED, estes podem tornar-se dispositivos mais eficientes que as lâmpadas convencionais. Realizaram um estudo comparando durezas avaliadas em uma resina composta polimerizada por cinco dispositivos baseados em LED (diodo emissor de luz com uma unidade de polimerização convencional. Todas as amostras fotopolimerizadas pelos dispositivos baseados em LED mostraram valores inferiores de dureza quando comparados com a lâmpada halógena no tempo de fotopolimerização típico (40 s). Concluiu também que tempos de exposição longos ou uma camada de resina mais fina são necessários para conseguir valores razoáveis da dureza em aparelhos baseados em LED.
Tsai et al. ${ }^{[5]}$ concluíram em seu trabalho que as luzes de polimerização LED disponíveis comercialmente usadas no estudo oferecem profundidade de polimerização e microdureza adequadas para espessuras de resinas que são recomendadas para uso no método de construção incremental $(2 \mathrm{~mm}$ ou menos). Para maiores espessuras de resinas, a performance das luzes de polimerização LED não é igual àquela das luzes de polimerização de quartzo tungstênio halógenas convencionais de alta intensidade.

Para se analisar a qualidade de resinas compostas, além dos ensaios de desgaste abrasivo, são efetuados ensaios de microdureza, entre outros. Ao realizar a análise in vitro de dureza, da contração de polimerização e da rugosidade de 29 resinas compostas, Carvalho Júnior ${ }^{[6]}$ concluiu que todas as resinas mostraram aumento da dureza e da contração de polimerização, ao se compararem os valores das três características obtidos para a medição imediata com os valores obtidos após 168 horas.

O objetivo deste trabalho de pesquisa científica, foi fazer uma comparação entre resinas poliméricas dentárias, polimerizadas por aparelhos baseados em lâmpada halógena e diodo emissor de Luz (LED), a tempos diferentes de polimerização, utilizando-se o método do disco retificado aperfeiçoado para odontologia e os respectivos valores de microdureza.

\section{Experimental}

Para avaliar o comportamento de resinas de cinco diferentes marcas quando fotopolimerizadas com o uso de aparelhos de fotopolimerização baseados em LED e em lâmpadas halógenas, a tempos diferentes, utilizou-se o método do disco retificado de Coelho ${ }^{[3]}$ com as modificações e adaptações desenvolvidas por Dias ${ }^{[7]}$ e Bianchi et al ${ }^{[2]}$.

Este método utiliza um banco de ensaios acoplado a um cabeçote de testes que possui um eixo para fixação de um disco agressor (disco dinâmico), que gira a uma rotação préestabelecida e proporcionada por um motor elétrico. O disco revestido com a resina (disco estático) a ser avaliado, é fixado numa parte do banco de ensaios, no qual também é fixada a mangueira por onde flui o líquido refrigerante e a carga aplicada. Nesta parte do banco de ensaios se tem um apoio, onde se coloca em contato o dispositivo que mede deslocamento. Este conjunto, apresentado na Figura 1, é posicionado sobre a mesa de uma retificadora tangencial plana, necessária à uniformização dos discos.

O disco dinâmico foi preparado por um protético experiente. Para tal realização, o disco dinâmico foi fabricado em prensa, através de um molde de aço inoxidável confeccionado para esta aplicação. A porcelana utilizada na fabricação deste disco foi da marca alemã Degussa ${ }^{\circledR}$, Duceran da cor C4. Este disco possuía um diâmetro de aproximadamente $26 \mathrm{~mm}$, necessário para posterior usinagem. Após a fabricação do disco dinâmico, foi necessária uma retificação até se obter um diâmetro de $24 \mathrm{~mm}$. Utilizou-se o rebolo de CBN (Nitreto Cúbico de Boro), da marca e filial brasileira Nikkon. 


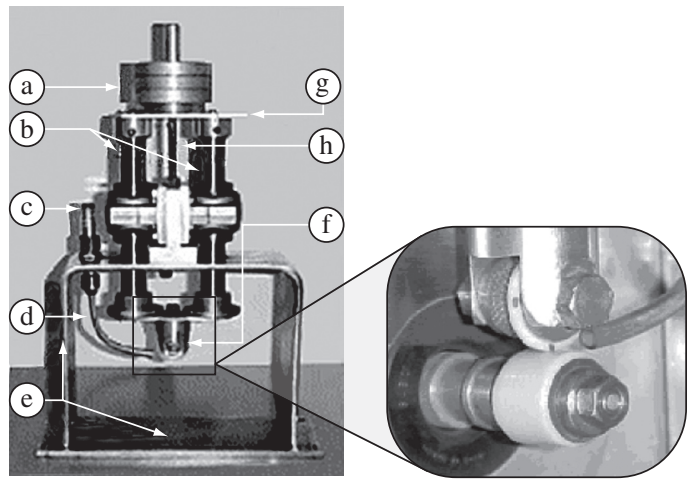

Figura 1. Banco de ensaios mostrado detalhes. a) Carga aplicada; b) hastes de deslocamento vertical; c) conexão para mangueira de refrigeração por água; d) Mangueira direcional do fluxo de água refrigerante ao contato entre os discos estático e dinâmico; e) suporte com base para apoio e fixação do dispositivo no cabeçote de testes; f) suporte com parafuso de fixação dos discos estáticos; g) apoio para o contato da esfera do apalpador eletrônico; e h) dispositivo de parada ("break").

Os discos estáticos (ou discos agredidos) foram recobertos com a resina. Utilizou-se um anel de aço ABNT 1020 usinado e em seguida, recartilhado em sua superfície externa para garantir uma melhor fixação das resinas, as faces laterais foram retificadas para que ficassem paralelas entre si, assegurando que todos tivessem a mesma medida.

Cada disco foi marcado pelo puncionamento de um código de letras utilizando punções. O revestimento foi formado pela polimerização de pequenos incrementos de resina depositados nessa região até que se recobriu toda a área. As resinas utilizadas neste estudo foram: Charisma, Suprafill, Fill Magic, Z100 E Tretic Ceram e os tempos utilizados para cada aparelho e cada amostra foram, 10, 20 e $40 \mathrm{~s}$. As resinas empregadas no estudo foram da cor A2.

Foram utilizados dois aparelhos polimerizadores para a fabricação das amostras de resinas. O primeiro dos aparelhos fotopolimerizadores utilizado foi o CL - K50, da empresa Kondortech. Neste aparelho a irradiação da fonte luminosa é feita através de uma lâmpada halógena com ponta de espessura de $8 \mathrm{~mm}$. O outro aparelho fotopolimerizador com polimerização através de LED foi Biolux Standard II com intensidade de luz de aproximadamente $100 \mathrm{~mW} / \mathrm{cm}^{2}$.

Após a regularização da superfície da resina (com um rebolo convencional de óxido de alumínio).

Após a confecção dos dois discos (dinâmico e estático), os discos foram instalados no banco de ensaios, no qual o disco fixo foi pressionado contra a superfície do dinâmico, sob uma força normal constante, através de uma carga préestabelecida de $16 \mathrm{~N}$.

A velocidade de rotação do disco dinâmico foi controlada através da freqüência do inversor para o funcionamento deste disco. Sua freqüência foi ajustada para $30 \mathrm{~Hz}$.

O ensaio foi iniciado com o contato entre o disco dinâmico e o disco estático. O desgaste da resina provocou o deslocamento $(\delta)$ do dispositivo, que foi medido através de um sensor eletrônico de deslocamento conectado a uma placa de aquisição de dados contida em um computador que, a partir de uma equação de calibração previamente implementada no programa de aquisição de dados (desenvolvido através do software Labview ${ }^{\circledR}$ ), gerou-se o gráfico de Deslocamento em função do tempo. Posteriormente, os dados coletados foram processados e analisados.

Para cada resina, a cada tempo, polimerizada por cada aparelho, foi feito um nível de repetibilidade de cinco ensaios. No total foram estudadas cinco diferentes marcas de resinas polimerizadas, cada uma, por dois aparelhos (LED e lâmpada halógena) e por três tempos diferentes de polimerização (10, 20 e 40 s), com exceção das resinas Charisma (polimerizadas somente por 10s e 20 s) e das resinas Suprafill (polimerizadas somente por 20 e $40 \mathrm{~s}$ ), obtendo-se um total de 26 corpos de prova.

Após o ensaio, os valores de tempo do gráfico de Deslocamento em função do tempo gerado pelo sistema de aquisição de dados foram elevados a 2/3 a fim de se obter uma reta, como é mostrado na Figura 2.

O gráfico mostra que no início não ocorreu deslocamento do dispositivo, porque se teve um período ocioso inicial entre

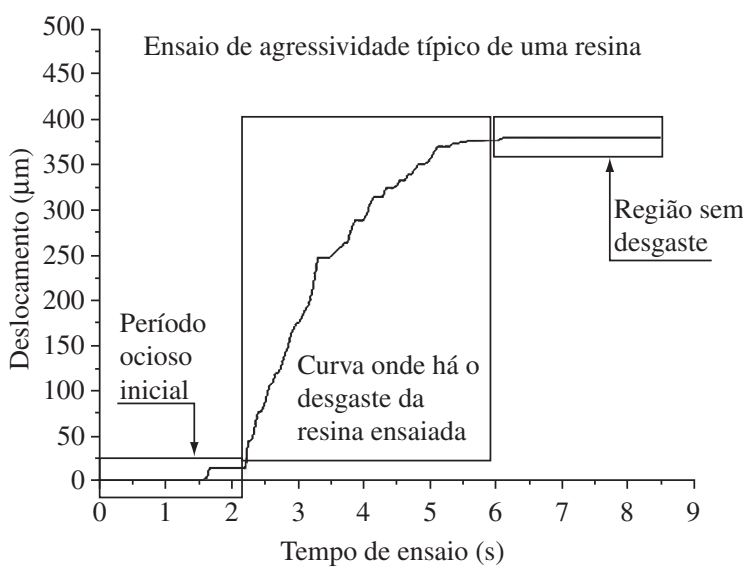

(a)

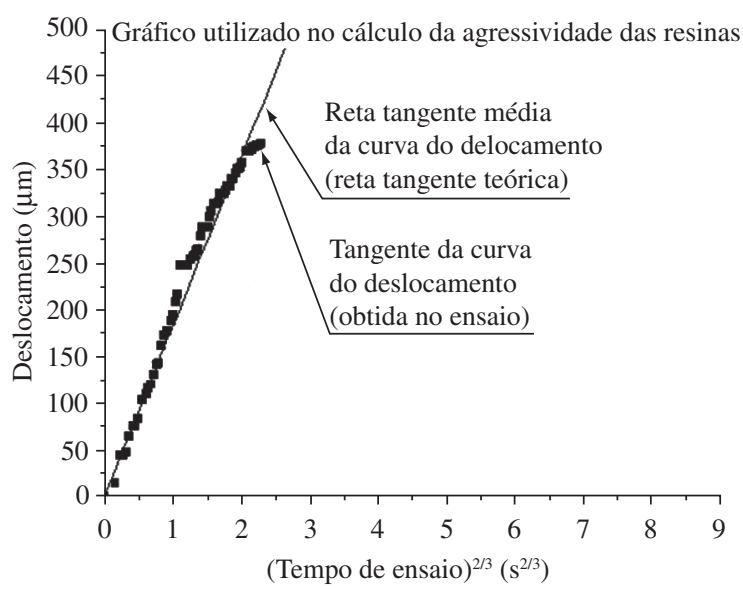

(b)

Figura 2. Gráficos mostrando os dados obtidos através do ensaio de desgaste das resinas. Em a) deslocamento em função do tempo; e b) deslocamento em função do tempo elevado a $2 / 3$. 
o acionamento do programa de aquisição de dados e o instante de acionamento do motor do cabeçote de ensaios. Como o medidor de deslocamento é muito sensível, conseguiu-se detectar mínimos sinais; assim, o primeiro deslocamento mostrado no gráfico não é um deslocamento real, visto que foi uma pequena variação de tensão da rede que foi registrada pelo sensor. Este primeiro instante foi desconsiderado.

Após esse instante, o ensaio de foi de fato realizado. Percebe-se uma curva crescente, que é apresentada no gráfico da Figura 2a. O cessamento do desgaste ocorreu devido ao ajuste de um dispositivo de parada, que impediu um maior desgaste da resina (constatado pelo comportamento constante ao final da curva da Figura 2a).

A única parte deste gráfico interessante para este estudo foi a correspondente ao ensaio, onde ocorreu o real desgaste da resina. O respectivo tempo de ensaio foi elevado a $2 / 3$, segundo a metodologia proposta por Coelho ${ }^{[3]} \mathrm{e} \mathrm{Dias}^{[7]}$. Ao se elevar o tempo a 2/3, como mostrado na Figura 3b, obteve-se um novo gráfico, este, uma reta. Então, fez-se uma regressão linear, onde se obteve o coeficiente angular (tangente) desta reta de aproximação. Através da Equação 1, determinou-se a agressividade $(\mathrm{k})$ da resina ensaiada, utilizando-se os parâmetros utilizados no ensaio (largura do disco estático $(\mathrm{b}=1,53 \mathrm{~mm})$, raio do disco estático para o respectivo ensaio (r) e força normal aplicada $\left(\mathrm{F}_{\mathrm{n}}=16 \mathrm{~N}\right)$ ).

$$
k=\frac{2 b \sqrt{4 r}}{3 F_{n}}\left(a_{1}\right)^{2 / 3}
$$

Sendo que $k\left[\mathrm{~mm}^{3} / \mathrm{N}\right.$.s $]$ é a agressividade da resina contra a porcelana; $F_{n}[\mathrm{~N}]$ a força normal necessária à remoção de material; $b[\mathrm{~mm}]$ a largura dos discos fixos; $r$ [mm] o raio dos discos e $a_{1}$ o coeficiente angular da reta de regressão linear.

Dessa equação tem-se que $\mathrm{a}_{1}$ maiores implicam em $\mathrm{k}$ maiores, mantendo os valores dos outros fatores. À medida que a resina desgasta mais rapidamente, a inclinação da reta de aproximação é maior, gerando um coeficiente angular maior. Logo maiores desgastes correspondem a maiores valores de agressividades das resinas contra a porcelana $(\mathrm{k})$, desde que os demais fatores sejam inalterados.

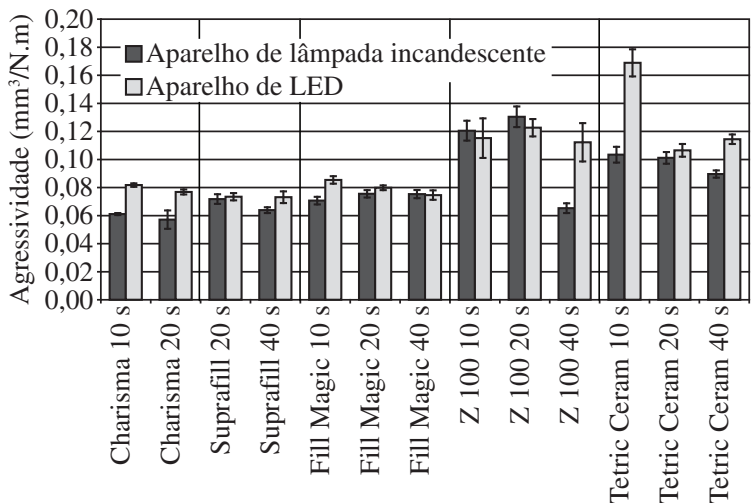

Figura 3. Resultados dos valores de agressividade obtidos nos ensaios com resinas.
O procedimento da realização dos ensaios de agressividade das resinas foi repetido por cinco vezes em cada amostra de resina. E assim, calcularam-se as médias dos valores de agressividade para cada amostra e também seu desvio padrão.

Foi realizada análise estatística ANOVA fator duplo, seguida do Teste de Tukey, com nível de significância de 5\%.

Além dos ensaios de desgaste, realizou-se também o ensaio de microdureza para cada corpo de prova. O equipamento usado foi o micro durômetro da marca BUEHLER, modelo 1600-6300.

A partir dos dados obtidos, tentou-se realizar uma correlação entre as análises de desgaste abrasivo com os valores de microdureza das resinas estudadas.

\section{Resultados e Discussão}

Com os valores coletados a partir dos ensaios de desgaste abrasivo, construiu-se o gráfico mostrados na Figura 3 que mostra todos os resultados de agressividade média e desvio padrão colhidos em laboratório, em um único gráfico, para uma melhor comparação entre os desgastes de cada uma. Estes valores foram submetidos à análise estatística pelo método ANOVA, ou análise de variância, e posteriormente ao teste de Tukey. Foram feitas comparações entre aparelhos, para cada tempo, de cada marca de resina.

O valor da agressividade é inversamente proporcional à resistência ao desgaste abrasivo. Assim, a resina que teve maior agressividade, sofreu maior desgaste abrasivo, sendo esta a menos resistente e a resina que teve menor agressividade, sofreu menor desgaste abrasivo, sendo, portanto, mais resistente ao desgaste. Desta forma a resina que apresentou maior agressividade, isto é, a que sofreu maior desgaste nos ensaios, foi a Tetric Ceram, polimerizada pelo aparelho de LED por $10 \mathrm{~s}$, cujo valor médio de agressividade obtido foi $0,170 \mathrm{~mm}^{3} /$ N.m., e a que sofreu menor agressividade foi a resina Charisma, polimerizada por lâmpada incandescente, por um tempo de $20 \mathrm{~s}$, cuja média nos valores de agressividade foram $0,057 \mathrm{~mm}^{3} / \mathrm{N} . \mathrm{m}$.

Os ensaios baseados no método do disco retificado demonstraram ser sensíveis e adequados para a realização das análises de desgaste das resinas compostas. Nesses ensaios, foram obtidos resultados que apresentaram diferentes estatísticas, com valores de desvios padrão satisfatórios.

O comportamento das amostras no ensaio de microdureza é apresentado no gráfico, representado na Figura 4, onde são mostrados os valores médios de microdureza Vickers, em HV, obtidos em laboratório e comparados os aparelhos fotopolimerizadores L.I. e LED, em cada amostra de resina, a cada tempo.

Os resultados com os valores de Microdureza também foram submetidos à análise estatística pelo método ANOVA, ou análise de variância, e posteriormente ao teste de Tukey. A análise foi realizada para comparar aparelhos entre si, em 


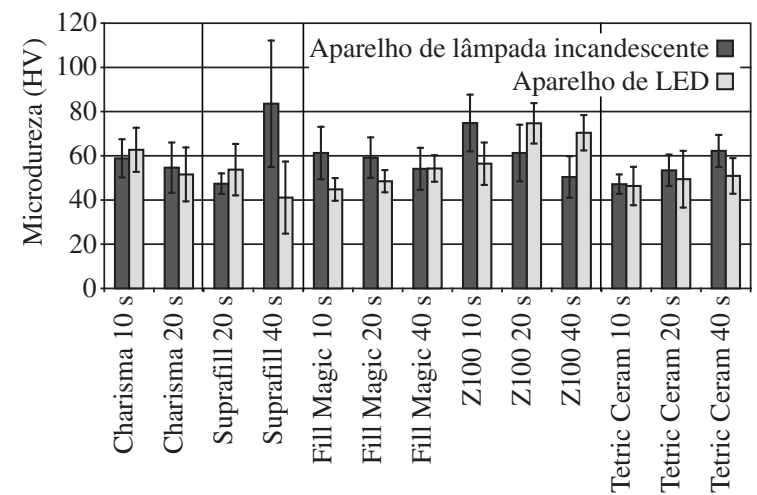

Figura 4. Gráfico com os valores médios da Microdureza das resinas comparando os aparelhos para cada tempo.

cada tempo de polimerização, em cada amostra de resina. $\mathrm{O}$ nível de significância foi de 5\%.

A resina que apresentou maior resultado de microdureza foi a Suprafill, polimerizada pelo aparelho de lâmpada incandescente por $40 \mathrm{~s}$, porém, seu desvio padrão foi relativamente elevado, obtendo-se um valor médio em seus resultados de microdureza de 83,56 HV, enquanto a resina que apresentou menor valor de microdureza foi a Suprafill, polimerizada por LED por 40s, com seu valor médio sendo 41,10 HV.

Porém, as análises estatísticas mostraram que numericamente, todos os resultados de microdureza são iguais. Não houve diferença estatística entre os resultados analisados, não sendo, portanto, um método seguro para se analisar o desgaste da resina através dele.

A comparação entre os dados obtidos nos ensaios de agressividade e nos ensaios de microdureza, respectivamente mostram que as resinas com maior desgaste (maiores valores de agressividade) não apresentaram necessariamente menores valores de microdureza. Isto pode ser comprovado observando os gráficos das Figuras 3 e 4. Isto se dá ao fato de as resinas compostas serem materiais que apresentam grande heterogeneidade, ou seja, trata-se de um material composto de partículas com durezas distintas e a microdureza varia de acordo com a dureza das partículas atingidas pelo indentador de medição de microdureza. Logo, os ensaios de Microdureza não demonstraram ser tão sensíveis para a realização das análises de desgaste das resinas compostas.

\section{Conclusões}

Através deste trabalho científico pôde-se avaliar a influência dos tempos de polimerização em resinas compostas polimerizadas com LED e Luz Incandescente, conforme foi proposto. Segundo a metodologia aplicada e dentro das condições experimentais do estudo conclui-se que, o ensaio de microdureza demonstrou não ser um método eficaz para determinar o desgaste abrasivo de resinas poliméricas dentárias, devido aos resultados apresentarem valores de desvios padrão relativamente altos, implicando em não se encontrar diferenças estatísticas entre as amostras. Isto ocorre em razão das resinas serem materiais bastante heterogêneos, que é de sua própria natureza.

$\mathrm{Na}$ análise estatística do desgaste abrasivo determinado pelo método do disco retificado, a resina que apresentou maior desgaste nos ensaios, foi a Tetric Ceram, polimerizada pelo aparelho de LED por $10 \mathrm{~s}$, cujo valor médio de agressividade obtido foi $0,170 \mathrm{~mm}^{3} / \mathrm{N}$.m., enquanto a resina com menor valor de agressividade foi a Charisma, polimerizada por lâmpada incandescente, por um tempo de 20s, cuja média dos valores de agressividade foi $0,057 \mathrm{~mm} / \mathrm{N} . \mathrm{m}$.

\section{Agradecimentos}

À FAPESP (Fundação de Amparo a Pesquisa do Estado de São Paulo) pelo apoio concedido.

\section{Referências Bibliográficas}

1. Dickinson, G. L.; Gerbo, L. R.; Leinfelder \& K. F. - Clinical evaluation of a highly wear resistant composite, Amer. J. Dent., 6, 2, p.85-7 (1993).

2. Bianchi, E. C.; Dias, A. C. P.; Bianchi, A. R. R. \& Freitas, C. A. - "Avaliação do desgaste abrasivo de resinas compostas", In: Anais do $4^{\circ}$ Congresso de Engenharia Mecânica Norte/Nordeste, Recife, 1996. . Rio de Janeiro, Associação Brasileira de Ciências Mecânicas, Vol.1, p.169-74 $\left(1996^{\mathrm{a}}\right)$.

3. Coelho, R. T. - "Estudo experimental da propriedade de dressagem de rebolos na retificação de precisão usando o método do disco retificado" Dissertação de Mestrado, Escola de Engenharia de São Carlos, Universidade de São Paulo, São Carlos, 122 p., 1991.

4. Kurachi, C.; Tuboy, A. M.; Magalhaes, D. V. \& Bagnato, V. S. - Hardness evaluation of a dental composite polymerized with experimental LED-based devices, Dental Materials, 17, 4, p.309-315 (2001).

5. Tsai, P. C. L.; Meyers, I. A. \& Walsh, L. J. - Depth of cure and surface microhardness of composite resin cured with blue LED curing lights, Dental Materials, Dental Materials, 20, 4, p.364-369 (2004).

6. Carvalho Junior, O. B. - "Avaliação "in vitro" da Dureza Superficial, da Contração de Polimerização e da Rugosidade de 29 Resinas Compostas", Tese de Doutorado, Universidade de São Paulo, Brasil, 2002.

7. Dias, A. C. P. - "Estudo e desenvolvimento de um método e um banco de ensaios para a avaliação do desempenho de resinas para restaurações odontológicas, assistido por computador" Monografia (Graduação), Faculdade de Engenharia e Tecnologia, Universidade Estadual Paulista, Bauru, Brasil, 143 p, 1995.

Enviado:04/10/06

Reenviado:13/03/07

Aceito: $19 / 04 / 07$ 